\title{
Chitosan and Cyclodextrin Modification on Cellulosic Fabric for Enhanced Natural Dyeing
}

\author{
M. SUNDRARAJAN*, R. RAJIV GANDHI, A. RUKMANI, S. SELVAM, \\ J. SURESH and S. GOWRI
}

Advanced Green Chemistry Lab, Department of Industrial Chemistry, School of Chemical Sciences, Alagappa University, Karaikudi -3, Tamil Nadu, India drmsgreenchemistrylab@gmail.com

Received 9 May 2012 / Accepted 28 May 2012

\begin{abstract}
Modification of the fabric is one of the best routes to improve the affinity between dye and fabric. In this study, chemical modification of organic cotton fabric has been done by the greener way with the use of $\beta$-cyclodextrin and chitosan and the modification level were measured by dyeing with natural dye (Nyctanthes arbor-tristis) parijataka. Results of dyeing showed better dye uptake after modification on organic cotton fabrics, especially in chitosan modification. SEM and FTIR studies reveals about the surface modification and the presence of $\beta$-cyclodextrin and chitosan moiety on the modified fabric. CCM studies of modified and unmodified fabric reported that chroma $\left(\mathrm{C}^{*}\right)$ values were found to be higher with modified fabric than unmodified fabric. Since the dyeability and other fastness properties of organic cotton are having better results. From an ecological point of view, organic cotton may be a better alternative to conventional cotton.
\end{abstract}

Keywords: Organic cotton, $\beta$-Cyclodextrin, Chitosan, Modification, Natural dyeing

\section{Introduction}

In dyeing field there is the growing interest to minimize the pollution problem, which is mainly caused by the synthetic chemicals. Presently there is an excessive use of synthetic dyes, which is estimated around 10,000,000 tones per annum. Conventional wisdom leads to the belief that natural dyes are friendlier to the environment than their synthetic counter parts. Natural dyes can exhibit better biodegradability and generally have a higher compatibility with the environment ${ }^{1-4}$. Natural dyes are obtained from substances such as flowers, trees, shrubs, buries, lichens, shellfish, leaves, insects and minerals ${ }^{5}$. Nyctanthes arbor-tristis is a valuable medicinal plant which belongs to the family oleaceae; it is a small tree with scented white flowers ${ }^{6}$. It contains coloring matter Nyctanthin which is identical with cracetin $\left(\mathrm{C}_{20} \mathrm{H}_{24} \mathrm{O}_{4}\right)$ from salfron ${ }^{7}$. Cotton is the abundant natural fibre which consist of practically pure cellulose (about $88-96 \%)^{8}$. High levels of agrochemicals are used in the production of non-organic, conventional cotton. 
Cotton production uses more chemicals per unit area than any other crop and accounts in total for $16 \%$ of the world's pesticides. Organic cotton is generally understood as cotton that is grown from plants without chemical fertilizers or pesticides which are not genetically modified, though organic cotton has less environmental impact than conventional cotton and it costs more for its production'.

Chitosan was proved to have the best chelating properties among other natural polymers ${ }^{10}$. Structurally, chitosan contains two main functional groups, namely hydroxyl and amino groups, as well as ether linkages. It was found that chitosan enhances the uptake of lac dye and increases lac dye sorption on cotton ${ }^{11}$. Cyclodextrins (CDs) are macrocyclic oligosugars most commonly composed of 6,7 or 8 glucosidic units bearing the names $\alpha-, \beta$ - and $\gamma$ - respectively ${ }^{12-14}$. The properties of cyclodextrins enable them to be used in a variety of different textile applications. Cyclodextrins may act as auxiliaries in washing and dyeing processes and they can also be fixed onto different fibre surfaces ${ }^{15}$. The permanent binding of CDs onto textile fibers offers the advantage that the inclusive properties of CDs become intrinsic to the modified fibers ${ }^{16}$. The purpose of this study is to improve the efficiency of dyeing in organic cotton fabrics with natural dye (orange corolla tubes of parijatak flowers) using the ecofriendly modifying agents such as $\beta$-cyclodextrin and chitosan.

\section{Experimental}

The fabrics used for the experimental work are bleached organic cotton (Scotts garments limited, Tirupur, India) in open dye bath. Parijatak flowers are the dye source and modification chemicals used are $\beta$-cyclodextrin, citric acid and chitosan which were prepared by using hydrochloric acid, sodium hydroxide and acetic acid. Sodium chloride and sodium carbonate salts were used as dyeing chemicals.

\section{Modification of organic cotton using $\beta$-cyclodextrin}

The modification process was carried at $80{ }^{\circ} \mathrm{C}$ for 60 minutes. The ML ratio was maintained carefully at 1:30 for modifications. $\beta$-Cyclodextrin $(0.75 \mathrm{~g} / \mathrm{L})$ and cross linking agent (citric acid $0.25 \mathrm{~g} / \mathrm{L}$ ) were used, after modification the samples were washed, dried and entered into dyeing process.

\section{Preparation of chitosan}

The shells of crab or prawn were washed well with sea water and again with fresh water before converting it into the final product. Crab shell was decalcified with dilute aqueous $\mathrm{HCl}$ solution. Chitin was produced by the deproteination of decalcified crab shells using dilute aqueous sodium hydroxide. Then the chitin was treated with hot concentrated $\mathrm{NaOH}$ solution for deacetylation to give chitosan.

\section{Modification of organic cotton using chitosan}

The fabrics were soaked in different concentration of chitosan solutions $(1 \mathrm{~g}, 2 \mathrm{~g}$ and $3 \mathrm{~g})$ and $2 \%$ of acetic acid concentration and 1:30 ML ratio were maintained for all the three modification baths. The samples were squeezed to a wet pick up of approximately $100 \%$. The samples were then dried in an oven at $85{ }^{\circ} \mathrm{C}$ for 5 minutes. Fabrics were rinsed with cold water and dried at room temperature for 24 hours. After modification, the samples were allowed to dyeing process. 


\section{Preparation of dye from parijataka flowers}

The botanical name of parijatak is Nyctanthes arbor- tristis. It is a short stalk flower with white petals and orange corolla tube. Only the orange corolla tubes were used for dyeing. The orange corolla tubes are shadow dried and grained well to get fine powder. This powdered form of dye was used for further dyeing process.

\section{Dyeing}

The dyeing process was carried out in two different conditions. The modified \& unmodified fabrics were entered into dye bath containing $5 \%$ of dye and $10 \%$ of sodium chloride, after 15 minutes $3 \%$ of sodium carbonate was added and stirred well by maintaining at $80{ }^{\circ} \mathrm{C}$ temperature. Finally the fabric was removed from the dye bath after 30 minutes and then the dyed samples were rinsed and dried. Similarly dyeing was carried out without salts like sodium chloride and sodium carbonate.

\section{Optical density [OD]}

The exhaustion values of dyed samples were measured by taking the absorbance of the dye liquor sample (before and after dyeing) using an UV-visible spectrometer. The optical density of original dye and spent dye liquor were observed and dye uptake was calculated using the following formula

$$
\text { Dye uptake }=\text { A1-A2 / A1 X } 100
$$

Where, $\mathrm{A} 1=\mathrm{OD}$ of original dye, $\mathrm{A} 2=\mathrm{OD}$ of spent dye liquor

\section{Washing fastness}

Washing fastness of all dyed samples was measured by the ISO 105-C03 testing method. Dyed samples were taken, stitched with one of the shorter side of the adjacent bleached fabric and put in to the bath containing $3 \mathrm{gpl}$ of soap, $2 \mathrm{gpl}$ of sodium carbonate and 1:30 MLR ratio at $60{ }^{\circ} \mathrm{C}$ for 30 minutes. Then the specimen was washed with hot water, cold water and dried.

\section{Instrumentation}

The exhaustion values of dyed samples were measured by taking the absorbance of the dye liquor sample (before and after dyeing) using an UV-visible spectrometer (Jasco V-530) at a wave length of maximum absorbance $\left(\lambda_{\max }\right)$ concerned. The characterization such as interactions and changes in chemical composition of modified and unmodified fabrics were measured by FT-IR [ $8400 \mathrm{~S}$ Shimadzu] $\left(4000-400 \mathrm{~cm}^{-1}, \mathrm{KBr}\right)$. To analyze the change in the surface morphology of modified and unmodified fabrics, we were examined with Scanning Electron Microscope (SEM) (Joel JSM8404) by gold coating sputtering method. The colour intensity of the dyed fabric was measured using a Computer Colour Matching (CCM) machine [V-2000 Konika Minalota] and the measurement was calibrated by a white tile and the colour values were measured taking barium sulphate as $100 \%$ reference standard, a piece of cloth was measured by using $\mathrm{D}_{65}$ light source with $10^{\circ}$ standard observer. Wash fastness of all dyed samples were measured by the (ISO 105-C03; 1989, Geneva), testing method.

\section{Results and Discussion}

Eco-friendly modification of organic cotton was carried out with $\beta$-cyclodextrin and chitosan to enhance the dye uptake of the natural dye parijataka. UV-Visible absorption spectra of dye solution showed the parts at blue region with a wavelength of $430 \mathrm{~nm}$ which confirms the appearance of yellow colour by parijataka dye as shown in Figure 1. 


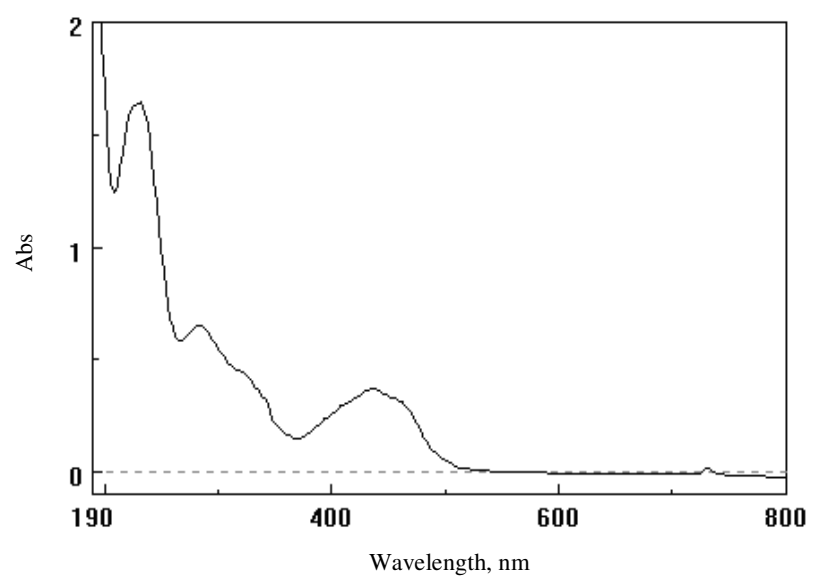

Figure 1. UV-Visible spectrum of Parijataka

\section{Modification of cellulosic fabrics with $\beta$-cyclodextrin and chitosan}

Modification of fabric with $\beta$-cyclodextrin was done at $80{ }^{\circ} \mathrm{C}$ using citric acid as a crosslinker to improve the dye uptake. $\beta$-cyclodextrin has the $-\mathrm{OH}$ functional group which was bonded to the fabric through the cross linker citric acid. Citric acid having a hydroxyl acid acts as a cross-linker between the fabric and the cyclodextrin. Since cyclodextrin has more $-\mathrm{OH}$ functional groups to react with dye molecule thereby it improves the dyeability. The cross linking of $\beta$-CD on the fabric was more effective and improved the dyeability. Modification with $\beta$-CD without citric acid is not effective because both fabric and $\beta$-CD has the same alcoholic functional group which repel each other hence it needs a cross-linker for bondage. Fabric was modified with chitosan to prepare various concentration solutions in $2 \%$ acetic acid $(1 \mathrm{~g}, 2 \mathrm{~g}$ and $3 \mathrm{~g})$. Among these, $2 \mathrm{~g}$ and $3 \mathrm{~g}$ concentrations of chitosan produced better dye uptake.

\section{CCM of modified and unmodified fabric}

Modified and unmodified fabric was dyed with and without salt and their CCM studies revealed that chitosan modified fabric produced better dye uptake than $\beta$-cyclodextrin. The $C^{*}$ values with regard to fabric type increased in the following order: 1 (Unmodified fabric dyeing without salt) $<2$ (Unmodified fabric dyeing with salt) $<3(\beta$-CD modified fabric dyeing without salt $)<4(\beta$-CD modified fabric dyeing with salt) $<\mathbf{5}$ (Chitosan modified fabric dyeing without salt) $<\mathbf{6}$ (Chitosan modified fabric dyeing with salt, it was clearly showed in Table 1.

Table 1. CCM values of dyed samples

\begin{tabular}{cccccc}
\hline Samples & $\mathrm{a}^{*}$ & $\mathrm{~b}^{*}$ & $\mathrm{~L}^{*}$ & $\mathrm{~h}^{\mathbf{0}}$ & $\mathrm{C}^{*}$ \\
\hline 1 & 0.6 & 2.5 & 1.45 & 3.5 & 1.02 \\
2 & 0.3 & 1.6 & 2.3 & 3.3 & 1.27 \\
3 & 0.5 & 0.5 & 2.2 & 2.46 & 1.82 \\
4 & 0.7 & 5.1 & 2.46 & 4.24 & 2.4 \\
5 & 0.4 & 0.8 & 4.2 & 6.46 & 2.8 \\
6 & 1.0 & 1.7 & 4.56 & 6.22 & 3.8 \\
\hline
\end{tabular}

$L^{*}=$ Light power, $a^{*}=$ Redder, $b^{*}=$ Greener, $h^{\circ}=$ Hue value, $C^{*}=$ Chroma value 


\section{FTIR and SEM studies of $\beta$-cyclodextrin and chitosan modified fabrics}

FTIR was used to examine the functional groups of the corresponding samples. Figure 3 [a-c] shows the IR spectrum of original, before and after modifications of the fabric. The observed transmittance peaks were presented in graph, the spectra of treated to untreated samples of organic cotton fabrics shows structural change, functional groups and existing groups. In chitosan modification, an additional double bond is formed which corresponds to the peak at $898.86 \mathrm{~cm}^{-1}$. FT-IR spectrum of $\beta$-cyclodextrin modified cotton showed peaks characteristic of $\beta$-cyclodextrin moiety. SEM image of unmodified fabric showed that the fibres are very flimsy but when modified with cyclodextrin and chitosan it looks dense due to the presence of the two moieties. Modification improved the porosity of the fabric which will enhance the dyeability (Figure 2).

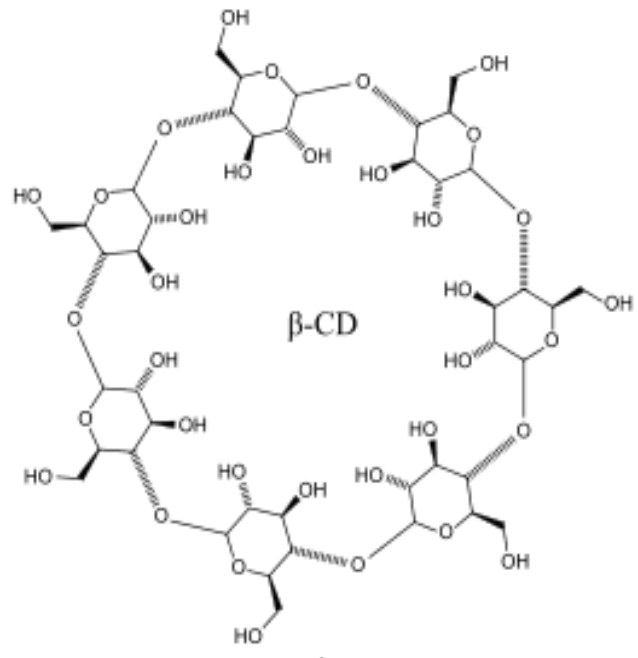

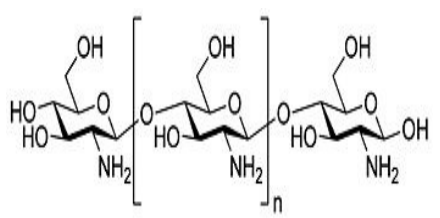

Structure of $\beta$-Cyclodextrin
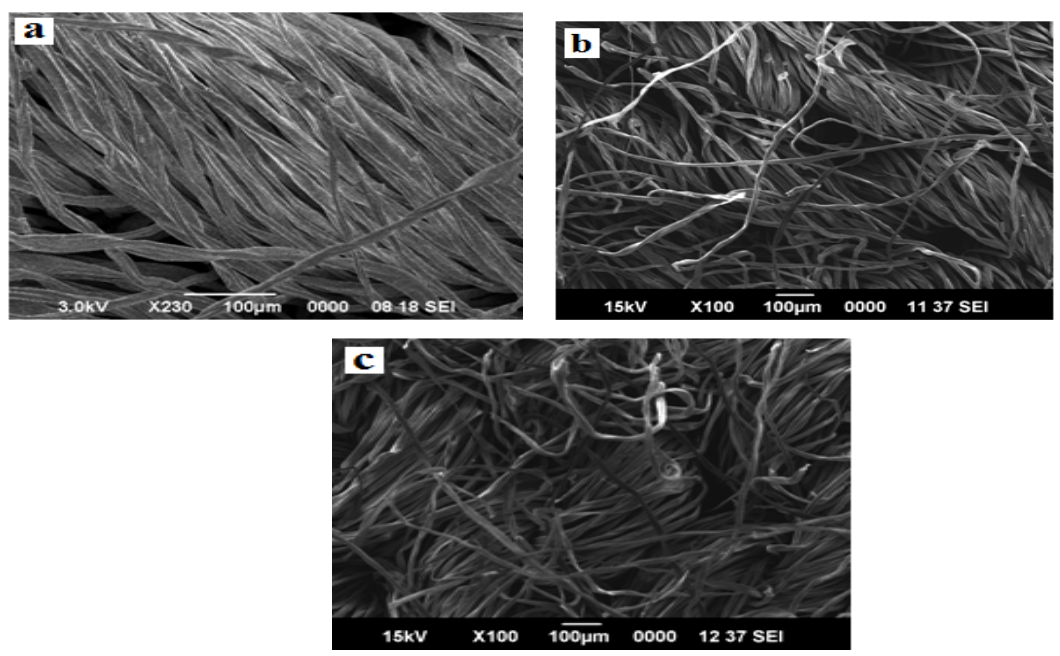

Figure 2. SEM images of (a) Unmodified organic cotton, (b) Cyclodextrin modified organic cotton, (c) Chitosan modified organic cotton 

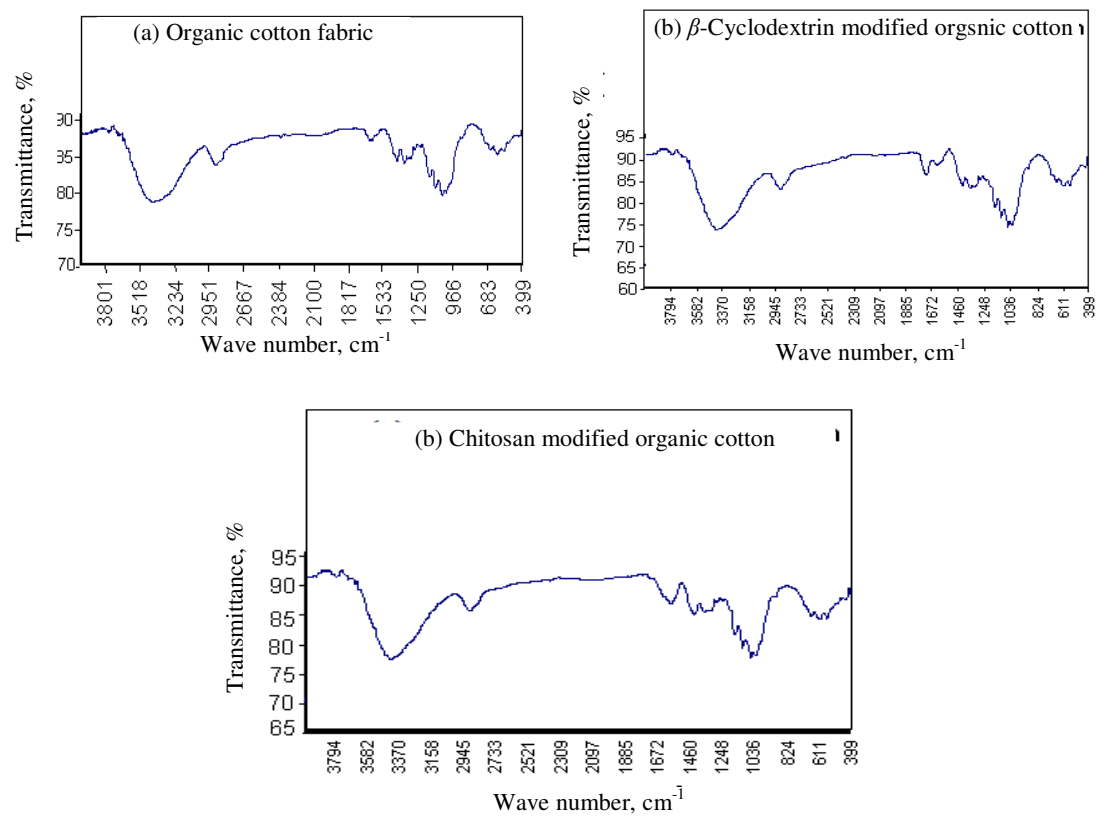

Figure 3. FT-IR spectrum of modified and unmodified fabrics

\section{Measurement of dye uptake and fastness properties of dyed samples}

Dye uptake comparison of $\beta$-cyclodextrin and chitosan modified organic cotton showed in Table 2. Organic cotton having good dye uptake values, it may be due to its purity and nature of texture. Out of the two modifications, chitosan produced better dye uptake. The amino group of chitosan was more reactive with dye molecule due to its cationic nature. But $\beta$ - $\mathrm{CD}$ has more $-\mathrm{OH}$ groups which are anionic and repelled to some extent by the anion of the dye molecule. It will be eliminated by using salts and fixing agents for dyeing. While comparing the appearance of dyed fabric, $\beta$-CD modified fabric showed bright yellow shade than chitosan modified fabric because of the difference in the reacting groups. The washing fastness of unmodified and modified fabrics with and without salts showed was shown in Table 2. The modified fabrics with salt were capable to retain the dye due to strong hydrogen bonding with the amino and hydroxyl groups of chitosan and cyclodextrin existing on the fabric. The poor fastness property of modified fabrics without salt may be due to weak interaction between the dye and fabric.

Table 2. Dye Uptake and Fastness range of dyed samples

\begin{tabular}{ccccccccc}
\hline & \multicolumn{3}{c}{$\begin{array}{c}\text { Without salt } \\
\text { SaD of Initial Liquor }=3.5)\end{array}$} & \multicolumn{4}{c}{$\begin{array}{c}\text { With salt } \\
\text { (OD of Initial Liquor }=5.0)\end{array}$} \\
\cline { 2 - 9 } & O.D & $\begin{array}{c}\text { Dye } \\
\text { uptake }\end{array}$ & $\begin{array}{c}\text { Washing } \\
\text { fastness } \\
\text { Shade }\end{array}$ & Stain & Dye & uptake & O.D & \multicolumn{2}{c}{$\begin{array}{c}\text { Washing } \\
\text { fastness } \\
\text { Shade }\end{array}$} & Stain \\
\hline $\begin{array}{c}\text { Un modified } \\
\text { fabric }\end{array}$ & 1.6400 & 53.14 & 3 & 2 & 2.2500 & 55.00 & 3 & 2 \\
$\begin{array}{c}\beta-C D \text { modified } \\
\text { fabric }\end{array}$ & 1.3475 & 61.50 & 4 & 2 & 1.7643 & 64.71 & 4 & 3 \\
$\begin{array}{c}\text { Chitosan } \\
\text { modified fabric }\end{array}$ & 1.2504 & 64.27 & 3 & 2 & 1.6246 & 67.50 & 4 & 2 \\
\hline
\end{tabular}




\section{Conclusion}

Modification of fabric was an essential criteria to improve their dye uptake and also to reduce the effluent load. If eco-friendly modification agents are used it will protect the environment from pollution. Hence eco-friendly biodegradable modification agent's $\beta$-CD and chitosan were used in this study to improve the dyeability of natural dye parijataka. $\beta$-CD and chitosan moieties are linked on the fabric thereby increased the dye uptake. But chitosan produced better result than $\beta$-CD because of the cationic nature of amino group present in it. FT-IR studies proved the presence of chitosan and $\beta$-CD moiety on the fabric. SEM analysis showed the surface morphology changes in the modified fabric. Therefore dyeing of an eco-friendly fabric, organic cotton with natural dye using biocompatible and biodegradable modification agents such as chitosan and cyclodextrin will be the cost effective environmental friendly approach in the field of dyeing industry.

\section{References}

1. Sudhakar R, Ningegowda K N and Padaki N V, Colourage, 2006, 53(7), 61-66.

2. Singh R, Jain A, Panwar S and Khare S K, Dyes and Pigments, 2005, 66(2), 99-102.

3. Han S Y and Yang Y, Dyes and Pigments, 2005, 64, 157-181.

4. Gupta D, Jain A and Panwar S, Indian J Fibre Text Res, 2005, 30(2), 190.

5. Siddhanta A K, Meena R, Prasad G, Mehta G K, Chhatbar M U, Oza M D and Sanjay Kumar, Naresh D S, Bioresource Technology, 2009, 100(24), 6669-6673.

6. Chairat M and Bremner J B Chantrapomma K, Fibres Polymers, 2007, 8(6), 613-619.

7. Thapliyal R C and Naithani K C, Seed Sci Technology., 1996, 24, 67-73.

8. Lewis Sr R J, Hawley's condensed Chemical Dictionary, $12^{\text {th }}$ Ed., Van Nostrand Reinhold, New York, 1993.

9. Haffegee J, Picking Cotton Carefully, Institute of Science in Society, March, 2007.

10. Varma A J, Deshpande S V and Kennady J F, Carbohyd.polym, 2004, 55, 77-93.

11. Saxena S, Iyer V, Shaikh A I and Shenai V A, Colourage, 1997, 44(11), 23-28.

12. Szejtli J, Comprehensive Supramolecular Chemistry, Pergamon, Oxford, 1996, 3.

13. Bender M L and Momiyama M, Cyclodextrin Chemistry, Springer, Berlin, 1978.

14. Ronald B, Steven D and Dong, Chem Rev., 1998, 98(5), 1997-2012.

15. Buschmann H J, Denter U, Knittel D and Schollmeyer E, J Tex Inst., 1998, 89, 554-561.

16. Sricharussin W, Sopajaree C, Maneerung T and Sangsuriya N, J Tex Inst., 2009, 100, $682-687$. 